\title{
The Field Of Lyman-Alpha Radiation plus Current Sheets and Physics of Different Zones of the Solar Atmosphere
}

\author{
Ostapenko V. A. \\ Academician of UAS, Ukraine, Kiev,
}

\begin{abstract}
The solution of the problem of the Lyman-alpha radiation field in the solar atmosphere simultaneously with discovery of the current sheets (CS) in the Nature (on the Sun) have allowed by the author to revise both the available models of solar atmosphere and the heat nature of the corona. We distinguish four zones now in the external solar atmosphere, in their sequence and in their physical sense: photosphere, transition zone, chromosphere, and corona. Now, it is believed that coronal plasma temperature has exceeded of million degrees. Our results indicate the structure of the corona composed of multiple non-thermal jets of the CS that change the very notion of the term of temperature. The corona is transparent in all hydrogen lines of the Lyman series (the A case of Menzel approximation) and radiative processes take only place in excitation of hydrogen plasma. In the solar atmosphere physical conditions of any of zones are determined, in other equal conditions, by the hydrogen plasma density only. The corona begins when the density of plasma will decreased to $n_{H}<10^{10} \mathrm{~cm}^{-3}$. The chromosphere occupied the plasma density diapason of $10^{10}<n_{H}<10^{14} \mathrm{~cm}^{-3}$. The transition zone occupied the plasma density diapason of $10^{14}<n_{H}<10^{18} \mathrm{~cm}^{-3}$. In this zone the LTE is achieved into all hydrogen lines of the Lyman series (the B case of the Menzel approximation) and electron impact processes take only place in excitation of hydrogen plasma. Here discrete lines of absorption are formed (the Fraunhofer spectrum of the Sun). And all conditions for the creation of multiple vertical CS with directed streams ejections take place because the plasma gradient is presence. And when the density $n_{H}>10^{18} \mathrm{~cm}^{-3}$, the continuous spectrum of the Sun arises, which is the radiation spectrum of negative hydrogen ions. In a vertical current sheet, the plasma density gradient causes a sharp decrease in the thickness of the compressed CS layer up to zero to the its middle and it turns the CS into the piston with one-sided eject up of the plasma. Multiple set of such accelerated plasma flows up and provides seemingly "heat" of the corona.
\end{abstract}

Keywords: the Sun, atmosphere zone, photosphere, chromosphere, transition region, corona, hydrogen Lymanalpha, current sheet, the corona heating, physics of solar zones.

\section{Introduction}

The observations of coronal loops, made from TRACE space observatory, show that the heating process is going on from below near the foot of loops where they connect to the solar surface. New observation results refute a general accepted theory which suggests equal heating of loops. The UV- images by the TRACE space observatory show bundles of heating up coronal loops. The discovery of current sheets in Nature is one of main results of our study (Ostapenko, 2011, 2012, 2016). This is a direct (first experimental) source of the heating up of corona that is formed in the transition zone of solar atmosphere. The new heating up mechanism of corona represents the nonthermal acceleration of charged particles and plasma as the result of magnetic force line reconnection in multitude of small current sheets in transition zone. In any case, plasma fluxes in the solar atmosphere are observed in a great quantity. Namely in our work has been shown that solar flare (in the chromosphere) as well as «moustaches» (in the transition zone) are by current sheets directly.

Solar flares of any magnificence are proven meet by the single approved the picture of the forming in the active region of the current sheet and the subsequent continuous increase of plasma density in the CS compressed layer. The stage, which develops each flare is the only achievement of the equilibrium magnetic and gas pressures in the $C S$ layer $\left(\boldsymbol{B}^{2} / \boldsymbol{8} \boldsymbol{\pi}=\boldsymbol{n}_{c s} \boldsymbol{k} \boldsymbol{T}\right)$. The evolution of any solar flare is the process itself of the magnetic fields collision. The CS compressed layer (weak flares; $n_{c s}<510^{16} \mathrm{cn}^{-3}$ ) is transparent for continuous radiation of the photosphere (csTRF flare) and the outside does not is detected for observers. The «black» flare stage ( $c s B L F$ - Black Light Flare) corresponds to intermediate values $\left(510^{16}<n_{c s}<710^{17} \mathrm{cn}^{-3}\right)$ of plasma density in the $C S$ layer and it precedes of the «white» flare $(c s W L F)$ when $n_{c s}>10^{18} \mathrm{cn}^{-3}$ (Ostapenko, 2011, 2012).

The adequate CS model is a laminar compacted layer of chromosphere plasma, geometric dimensions of which do not exceed the size of the arch system of new magnetic flux. The calculated plasma density $\mathrm{N}_{\mathrm{cs}}$ and observed ejection velocity of plasma flows $\mathrm{V}_{\mathrm{cs}}$, as also as the effective strength of magnetic fields $\boldsymbol{B}$ in the contact area of new and existing fields are connected each other by expression: $B=V_{c s} \sqrt{4 \pi \rho}=V_{c s}$ 
$\sqrt{4 \pi m_{H} N_{c s}}$. This allows us to link between the flare score and the strength of magnetic fields providing the magnetic classification of flares on magnitude. The external manifestation of CS (fig.4) in continuous radiation is determined only by the $\mathrm{N}_{\mathrm{cs}}$, in other words, only the magnitude of the magnetic fields. The plasma density $\mathrm{N}_{\mathrm{cs}}$ is increased with chromosphere density $10^{13} \mathrm{~cm}^{-3}$ up to $2-510^{18} \mathrm{~cm}^{-3}$ (with the small $\mathrm{L}_{\mathrm{cs}} \sim 10^{5}-10^{1} \mathrm{~cm}$ ). The magnetic field at each flare is various. Therefore, there are transparent in a continuous spectrum (csTRF), black (csBLF) and white (csWLF) flares of different shapes and sizes.

\section{The Lyman-alpha $\left(L_{\triangleright}\right)$ Radiation Of Hydrogen Plasmas}

The temperature $T_{21}$ of $L_{\alpha}$-radiation is important to know when physical conditions in the emission formations are found by the solution method of UST equations system of the statistical equilibrium. This is the problem of assessing the one of main parameters of the model, which is the plasma density. When the temperature is $<8000 \mathrm{~K}$ the majority of hydrogen atoms is located in the ground quantum state. All transitions on the first level are accompanied by radiation in the $U V$ region and for ground-based observer unachievable. At the same time, the relationship between the electron temperature $T_{e}$ and temperature $T_{2 I}$ is unknown. Any researcher must to consider of the $T_{21}$ as a free parameter (Sobolev, 1958, 1961). Of course, in such cases, we may to use the two extremely approximations. In first, this is the $A$ case - optical thicknesses is zero in the Lyman series; Secondly, this is the $B$ case - the LTR conditions are in the lines of the Lyman series (Baker, Menzel, 1938; Seaton, 1959). In our calculations is used the hydrogen atom model with the levels number $m=9$ and continuum. The density $\left[n_{H}, \mathrm{~cm}^{-3}\right]$ of hydrogen atoms

$n_{H}=n_{e}+\sum_{m=1}^{\infty} n_{m}$,

The source parameters are $n_{H}, T_{e}$ and $l$ (the length of the object along the line of sight), as well as the Doppler width $\Delta \lambda_{D} / \lambda=0.710^{-4}$. The temperature $L_{\alpha}$ - radiation can be found from the stationary equation for the first level:

$$
\frac{N_{2}}{N_{1}}=\frac{n_{e} C_{1 c}+n_{e} \sum_{m=2}^{M} C_{1 m}+B_{1 c} J_{1 c}^{\oplus}+\sum_{m=2}^{M} B_{1 m} J_{1 m}^{\oplus}}{n_{e} \sum_{m=2}^{M} \frac{N_{m}}{N_{2}} C_{m 1}+\sum_{m=2}^{M} \frac{N_{m}}{N_{2}} A_{m 1} \beta_{1 m}+\frac{n_{e}^{2} l}{N_{2}}\left[A_{c 1} \beta_{1 c}+n_{e} C_{c 1}\right]} .
$$

Here $C_{1 m}$ and $\mathrm{C}_{1 \mathrm{c}}$ are the excitation and ionization coefficients of the hydrogen atoms by electron impacts. Numerical values of the coefficients are given in Ostapenko (1974). The $A_{m l}$ and $B_{l m}$ are the Einstein ratios for radiative transitions. The number of transitions in the result of the excitation by external radiation field is calculated according to the formula (Chultem \& Yakovkin, 1974)

$N_{l} B_{l m} J_{1 m}^{\oplus}=I_{1 m}^{\oplus} W \beta_{1 \mu} \tau_{1 \mu} \mu_{1 \mu}$,

$\mu_{1 \mu}=0.11210^{18} \frac{\Delta \lambda_{D}}{\lambda} \lambda^{2}$, and $\mathrm{W}$ is dilution factor.

To calculate the probability of outlet photons used approximate expression. The $N_{1} B_{l c} J_{1 c}^{\oplus}$ coefficient is presented in the same form as (3). So, the amount of solar energy that is absorbed in the continuum can be written:

$E_{a b s}=\int_{\lambda_{0}}^{0} I_{\lambda}\left(1-e^{-\tau_{\lambda}}\right) d \lambda=I_{v_{0}}^{\oplus} \frac{\exp \left(\frac{h v_{0}}{k T^{\oplus}}\right)}{v_{0}^{3}} \int_{v_{0}}^{\infty}\left(1-e^{-\tau_{v}}\right) v^{3} \exp \left[-\frac{h v}{k T^{\oplus}}\right] d v$.

Using the expression for the quanta outlet for continuum, we get the photons number:

$N_{l} B_{l c} J_{1 c}^{\oplus}=\frac{E_{a b s}}{h v_{1 c}}=0.29710^{11} I_{\lambda_{0}}^{\oplus} \lambda_{0}^{2} \tau_{l c} \mu_{l c}=\mu_{l c} I_{\lambda_{0}}^{\oplus} \tau_{l c} \beta_{l c}$.

Photon energy $h v_{l c}$ is taken in some average value for $\lambda=850 \mathrm{~A}$. The number of escape photons in the Lyman continuum, with the required here precision, appears in the form:

$$
n_{e}^{2} l A_{c l} J_{l c}=\frac{E_{r a d}}{h \bar{v}_{1 c}}=0.12310^{-11} \frac{n_{e}^{2} l}{\sqrt{T_{e}}} \beta_{l c} \text {. }
$$


The population of the second level of the any flare powers remains the same (Krivsky \& Kurochka, 1974). The populations of the upper levels are little influenced on the results of the solution of the equation for the first level equilibrium.

To simplify of calculations, the ratios $N_{m} / N_{2}$ of population levels are described by the Boltzmann formula with the $T_{e x}=6000 \mathrm{~K}$ temperature (Kurochka et al., 1975). To fainter emission formations $\left(T_{e x}<6000 \mathrm{~K}\right)$, level populations above the third virtually no effect on the $N_{2} / N_{l}$. The $n_{e}$ is found from the equation of ionization equilibrium

$n_{e} \sum_{m=1}^{M} N_{m} C_{m c}+\sum_{m=1}^{M} N_{m} B_{m c} J_{m c}^{\oplus} W=$
$=n_{e}^{2} l\left[A_{c l} J_{l c}+\sum_{m=2}^{M} A_{c m}+n_{e} \sum_{m=1}^{M} C_{c m}\right]$,

As well as of equations

$$
\begin{aligned}
& \frac{n_{e}^{2}}{n_{2}}=\frac{2\left(2 \pi m_{e} k T_{e}\right)^{3 / 2}}{g_{2} h^{3}} \exp \left[-\frac{h v_{2 c}}{k T_{2 c}}\right], \\
& n_{2}=4 n_{1} \exp \left[-\frac{h v_{12}}{k T_{21}}\right], \quad n_{H}=n_{e}+n_{1} \text {. }
\end{aligned}
$$

If solve the system of equations (8) against $n_{e}$, we get

$$
n_{e}=\frac{\mathrm{X}(T)}{2}\left[\sqrt{\left(1+\frac{4 n_{H}}{\mathrm{X}(T)}\right)}-1\right], \quad \mathrm{X}(T)=\frac{\left(2 \pi m_{e} k T_{e}\right)^{3 / 2}}{h^{3} \exp \left[\frac{h v_{12}}{k T_{21}}+\frac{h v_{2 c}}{k T_{c 2}}\right]} \text {. }
$$

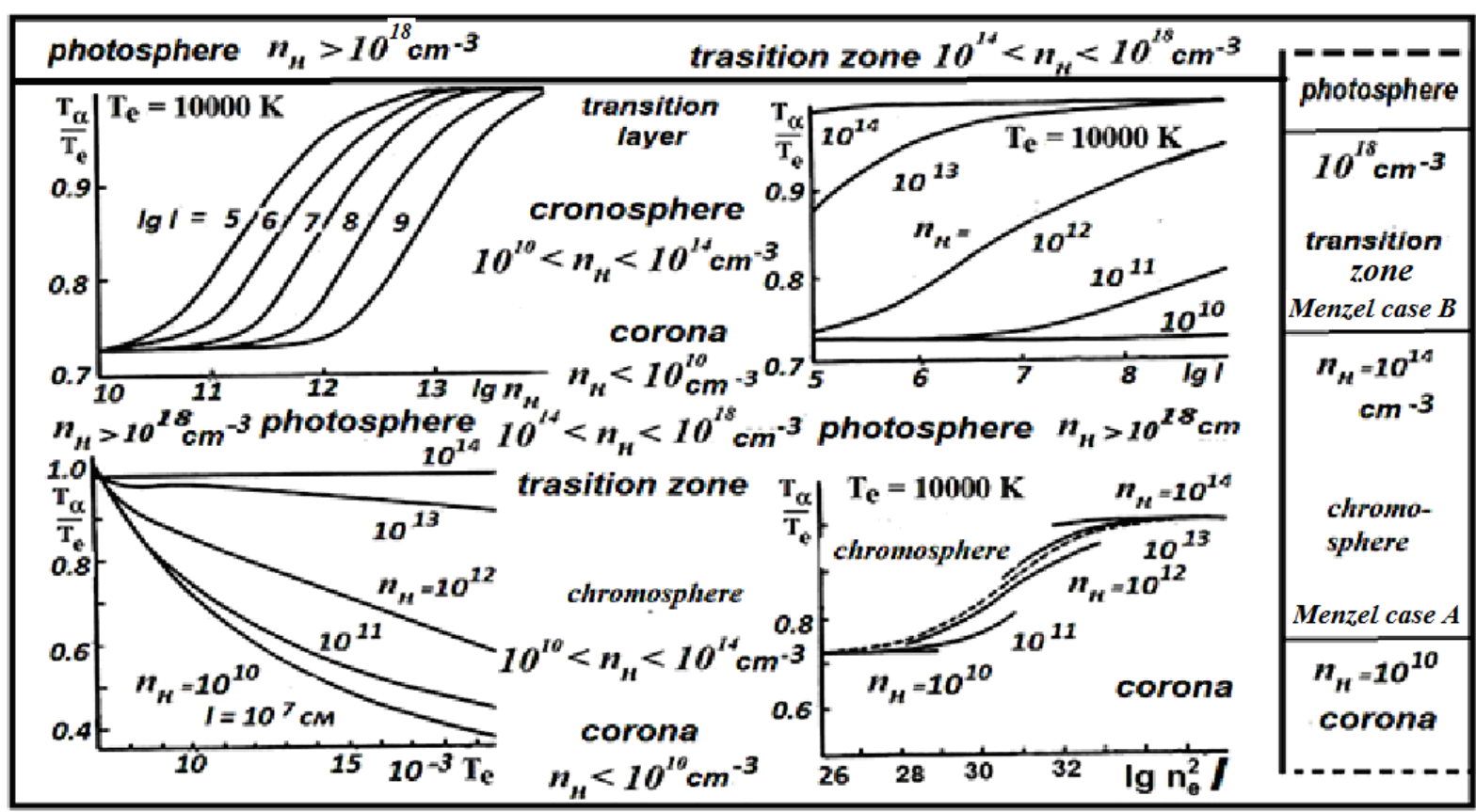

Fig.1. The definition of the physical sense of various zones in the atmosphere of the Sun.

These are photosphere, transition zone, chromosphere, and corona.

The curves show the Lyman-alpha $1216 \AA$ line of radiation field for hydrogen plasma in the Sun atmosphere in accordance of Kurochka et al. (Ostapenko, 2011, table 4.3.1).

The $n_{e}$ values are being found of the (7) \& (9) equations by the sequential approximation method. The $n_{e}$ first value is obtained in $T_{e}=T_{21}$ supposition as well as in supposing that the hydrogen ionization is caused by Balmer Sun's continuum (with $T_{2 c}=5600 \mathrm{~K}$ ). Substituting the obtained $n_{e}$ in the (2) equation, we find the first approximation of $N_{2} / N_{l}$. The new $T_{21}$ approach is used to obtain a new $n_{e}$ approach. The $T_{21}$ calculation results are given in Ostapenko (2011, table 4.3.1) for set parameters of $n_{H}, T_{e}$ and $l$ (Kurochka et al., 1978). Note that amongst the chromosphere formations was not such situations, where condition $T_{e}=T_{21}$ would is installed (fig.1). 
The LTE conditions (Menzel' case B) are only achieved in the transition zone at the $n_{H}>10^{14} \mathrm{~cm}^{-3}$ where, as is well known, the Fraunhofer lines are formed. The second extreme case (Menzel' case $A$ ), when optical thicknesses in the Lyman-alpha line of the are very small $\left(n_{H}<10^{10} \mathrm{~cm}^{-3}\right)$, the solar atmosphere becomes by the corona.

In the full UST equations system is conveniently used the (10) approximate expression, which has replaced the equation for the first level (for solar structures):

$$
\begin{aligned}
& T_{2 l}=7300+\frac{T_{e}-7300}{1+e^{-x}}, \\
& x=\lg n_{e}^{2} l-29.4-3.14\left(\lg T_{e}-3.7\right) .
\end{aligned}
$$

Fig. 1 shows that the LTE is always absent in chromosphere formations. The obtained results for the first time have given a quantitative definition of the different (physically reason now) zones of the Sun's atmosphere. So, the transition zone is begun after plasma density achieving of $n_{H} \sim 10^{14} \mathrm{~cm}^{-3}$. Here the photosphere absorption metal lines are formed and the local TDE as the case $B$ of the Menzel approximation is achieved in $L_{\alpha}$ hydrogen line (Baker, Menzel, 1938). The photosphere actually begins by achieving the $n_{H} \sim 10^{18}$ $\mathrm{cm}^{-3}$ plasma density. The hydrogen plasma is not transparent in the continuous spectrum during the negative hydrogen ion excitation (fig.4).

\section{Vertical Current Sheets In The Transition Zone}

The simultaneous calculations of lines emission of hydrogen atoms and the continuous emission of hydrogen negative ions as well as the flares spectra MF4A photometry have shown:

The $C S$ is transparent (in $c s T R F$ view) for photosphere continuous radiation as plasma densities in the $C S$ layer of $N_{c s}<510^{16} \mathrm{~cm}^{-3}$. The own continuous emission of flares is appeared of the first as a «black» $(\operatorname{csB} B F)$ flare when $510^{16}<N_{c s}<510^{17} \mathrm{~cm}^{-3}$ and, later, on sometimes, and a «white» $(c s W L F)$ flare in $510^{17}<N_{c s}<210^{18} \mathrm{~cm}^{-}$ 3 occurs in the $C S$. It displays the single $c S(T R F-B L F-W L F)$ process by progressively increasing of plasma density from the chromosphere density $\left(\sim 10^{13} \mathrm{~cm}^{-3}\right)$ until the arising of the turbulence stage and destruction of the $C S$ at $210^{18} \mathrm{~cm}^{-3}$.
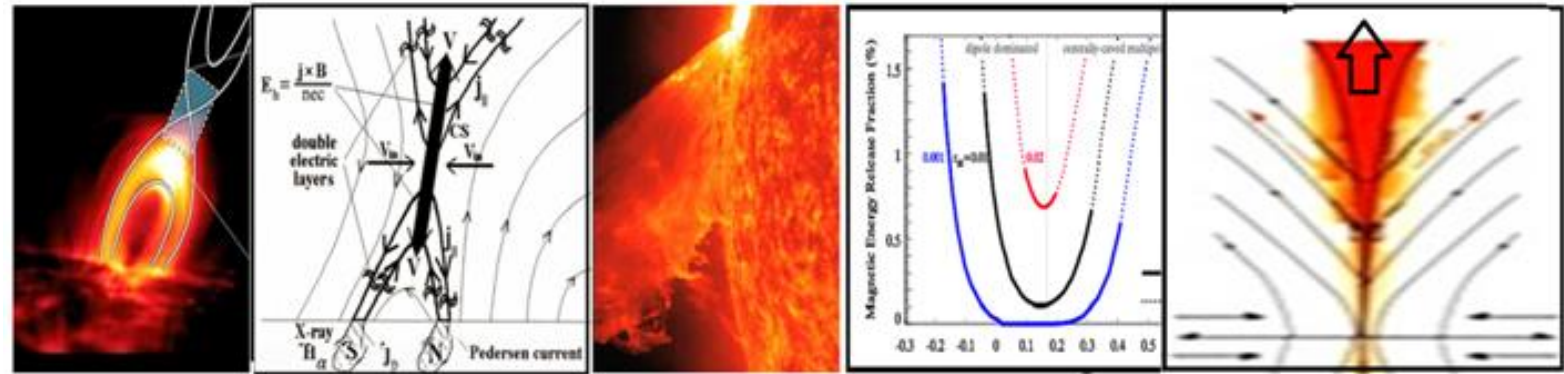

Fig.2. The symmetrical current sheet (CS) transforms into the piston in the transition zone.

a. The CS in the top of arch systems of active region (Burch \& Drake, 2009).

$\boldsymbol{b}$. The model of a vertical CS in the corona (Podgorny \& Podgorny, 2006).

c. The video film of the eruptive prominence (APOD-07.03.2011-NASA/Goddard/SDO-movie AIA Team).

$\boldsymbol{d}$. A twisted flux and a current sheet (CS) turns the CS into the piston with one-sided eject up of the plasma (modeling of Lei Huang \& Cong Yu, 2014).

$\boldsymbol{e}$. In the transition zone as rule and in the chromosphere often the gradient of plasma density turns the vertical CS into a piston with one-sided ejection upward of the plasma (mofelling and photometry, fig.3).

Lei Huang \& Cong Yu (2014) propose a physical mechanism to explain giant flares and radio events in terms of a magnetospheric model containing both a twisted flux and a current sheet (CS). With the appearance of CS, they solve a mixed boundary value problem to get the magnetospheric field based on a domain decomposition method. They investigate properties of the equilibrium curve of the flux when the CS is present in background of multipolar fields. In response to the variations at the magnetar surface, it quasi-statically evolves in stable equilibrium states. The loss of equilibrium occurs at a critical point and, beyond that point, it erupts catastrophically. New features show up when the CS is considered. They find two kinds of physical behaviors, i.e., catastrophic state transition and catastrophic escape. Magnetic energy would be released during state transitions. The released magnetic energy is sufficient to drive giant flares. The flux rope would go away from the magnetar quasi-statically, which is inconsistent with the radio afterglow. Fortunately, in the latter case, i.e., the catastrophic escape, the flux rope could escape the magnetar and go to infinity in a dynamical way. This 
The Field Of Lyman-Alpha Radiation plus Current Sheets and Physics of Different Zones of the ..

is more consistent with radio afterglow observations of giant flares. They find that the minor radius of flux rope has important implications for its eruption. Flux ropes with larger minor radius are more prone to erupt. Lei \& Cong (2014) stressed that the CS provides an ideal place for magnetic reconnection, which would further enhance the energy release during eruptions.

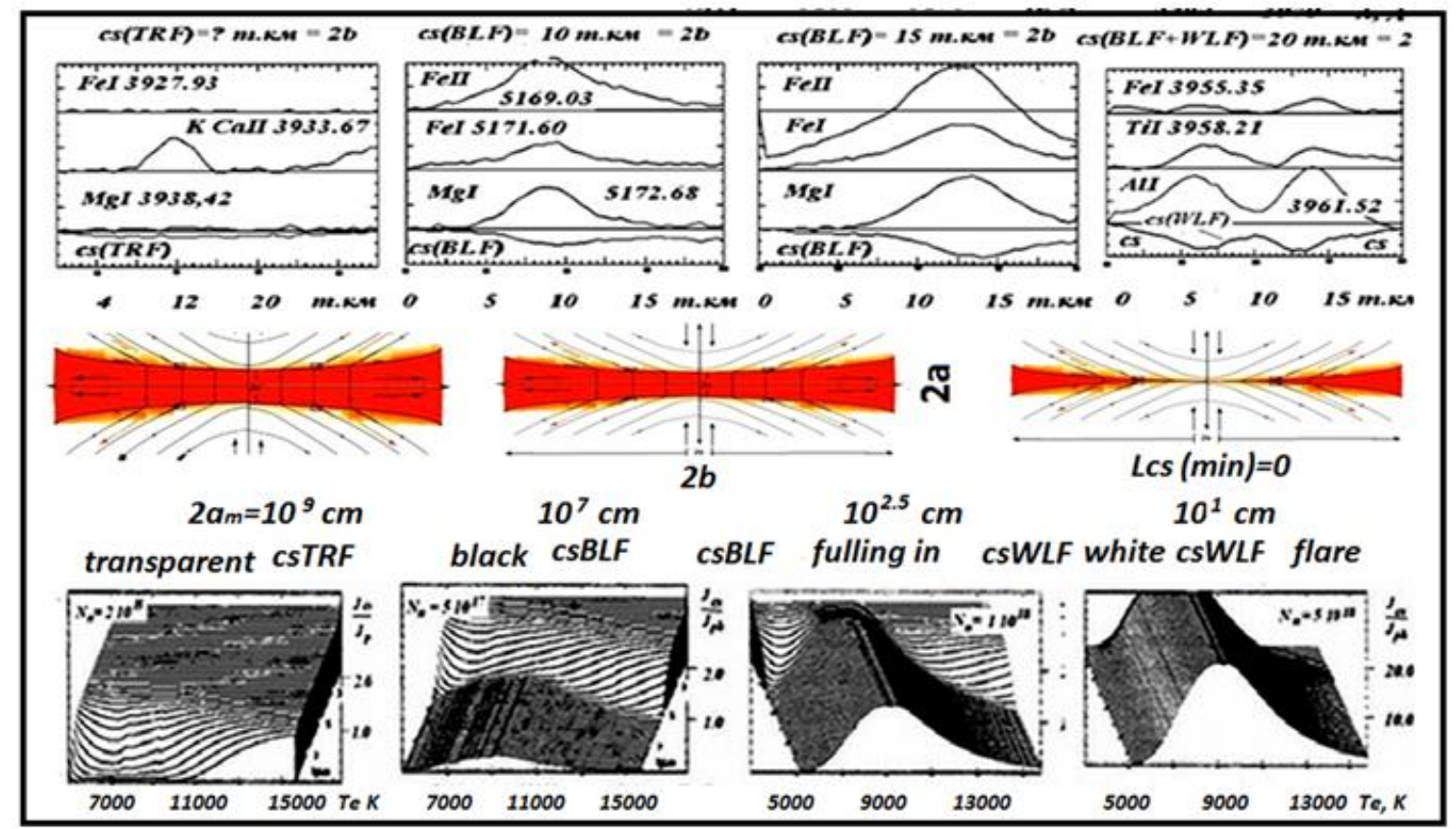

Fig.3. The cs $(T R F-B L F-W L F)$ development of the current sheet with the change of the flares power (Остапенко, 2011). In top panel are the flares spectra by the MF4A photometry along the spectral line (along the surface of the Sun). The CS layer width $2 b$ is increased while its thickness $2 a$ and is decreased with increasing of flares power. The maximal plasma density it is appeared to be in the middle of symmetrical layer (when the plasma gradient is absent) and reduced to chromosphere densities on its periphery.

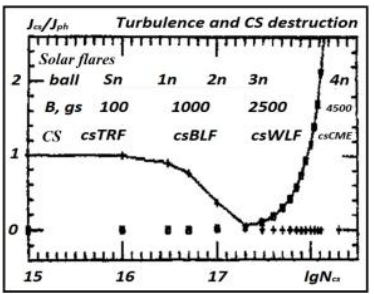

Fig.4. Changing of the external manifestation of the CS with increasing of the plasma density $N_{c s}$ from $10^{15}$ to $510^{18} \mathrm{~cm}^{-3}$ for $L_{c s}=10^{3} \mathrm{~cm}$ (by the 3D variant of fig.6.10.1). Observations are allowed to link the observed values of $V_{c s}$ and the calculation of $N_{c s}$ by flares of various powers or by strengths of interacting magnetic fields. The class $c s C M E$ is by flares of strongest power.

A geometric $C S$ thickness changes in the evolution process of the $C S$ compacted layer depth from $L_{c s}$ $\sim 10^{5} \mathrm{~cm}$ to the layer depth of the most powerful flares $L_{c s} \rightarrow 0 \mathrm{~cm}$ (that results the $C S$ destruction). Namely the process of sequential increase of the $C S$ plasma density displays the dynamic collision of magnetic fields and represents the $c s(T R F-B L F-W L F)$ evolution of any flare. The ended stage of the flare evolution and the largest $C S$ plasma density are defined only by strengths of magnetic fields. The importance classification of solar optical flares is, therefore, the magnetic classification. The picture is modified in near photosphere vertical CS when plasma gradient take place and the CS is transformed into a one-side piston (fig.2e).

The $C S$ development is shown in spectra examples of two strong disc flares on 15.07 .1981 and 09.07.1982 (Ostapenko, 2011). Calculations (fig.3) highlighted the following points. The CS layer extends further along the Sun than it is seen on the recording $c s B L F$ strips (top panel in fig.3). Indeed, the emission lines of metals observed wider along the surface of the Sun. According to our calculations (fig. 4), the current sheet is still presented in these places. It is also characterized by higher temperature, but has less value of plasma density because of the continuous $c s B L F$ radiation is not observed. Plasma density, therefore, varies along the transverse cut of the CS. The symmetrical CS is minimal at the CS periphery and the maximal in the middle area.

We explained why the hydrogen $H_{\alpha}$ line wings reach of $20 \AA$ widths at the moment $c s B L F$ appearance, and why they have achieved the $40 \AA$ widths and have disappeared together with same narrow lines of metals in the background of the $c s W L F$ appearing. The metals occur in a stable compacted CS layer and there are until the appearing of the $C S$ turbulence in significant magnetic fields on the $c s W L F$ background. The widths of hydrogen and $H \& K C A I I$ lines are increased with the growing of flare powers and they are depend on the speed of 
The Field Of Lyman-Alpha Radiation plus Current Sheets and Physics of Different Zones of the ..

throwing CS plasma flows, which are a function of the magnetic field strengths $\left(\boldsymbol{B}^{2} / 4 \pi \boldsymbol{m}_{H} \boldsymbol{N}_{c s}=V_{c s}{ }^{2} . \rightarrow \boldsymbol{V}_{c s}{ }^{2}=\right.$ $B^{2} / 4 \pi m_{H} N_{c s}=A N_{c s}$.). The appearing of the plasma turbulence as the data of photometry and our calculations show in fig.3,4) means the CS own destruction. The initial of the 15.07.1981 flare is associated with emergence of the new magnetic flux. This process is associated with the force lines destruction. The 15.07.1981 and 09.07.1982 disc flares are the basis of discovering of the current sheets on the Sun.

\section{The Sun's Internal And External Zones}

Internal layers: Like all the other main-sequence stars the Sun produces energy through the thermonuclear synthesis converting hydrogen to helium. The core in the center of the Sun where the temperature is $\sim 15$ million ${ }^{\circ} \mathrm{K}$ (for hydrogen cycle) is the region where nuclear synthesis takes place. Each second, in the Sun annihilates $>4$ million tones of hydrogen into helium. This internal energy is the source of energy of all released types of external solar energy.

In the currently theoretical solar model («the Standard Model», fig.5a), 98,5\% of energy is produced in the reactions of direct synthesis of helium from hydrogen. And only $1.5 \%$ of energy is produced in the reactions of the so-called $\mathrm{CNO}$ cycle, in which carbon is cyclically transformed into nitrogen and oxygen and then again a new cycle reaction leads to the creation of carbon. The mergence by four protons into $\alpha$-particle is accompanied by a general energy release of $26.732 \mathrm{MeV}$ that equals by the mass defect during the transformation of two protons into two neutrons. Two neutrinos, taking away $\sim 0.5 \mathrm{MeV}$, freely leave the Sun, the rest energy transforms into $\gamma$ - quanta and thermal energy of plasma. The source of this energy is the energy of the connection of the ${ }^{4} \mathrm{He}$ nuclei that corresponds to a mass defect of $0.73 \%$ by mass of four unbound protons.

Reaction of carbon cycle is the main energy source of more hot stars but regarding the Sun these reactions ensure $<1-2 \%$ of solar luminosity. Such reactions take place only in the center of the Sun where the temperature and density reach their maximum. When it goes away from the solar center, temperature and density become lower, the energy release as a result of carbon cycle stops quickly and on the distance of $0.2-0.3$ radius from the center only proton-proton chain reactions are left.

On the distance of $r>0.3$ from the solar center the temperature is about $<5 \cdot 10^{6} \mathrm{~K}$, significantly decreases and density. Nuclear reactions are stopped. The zone of radiative transfer $\left(0.3-0.7 R_{\Theta}\right)$ have carried only the radiation up to the surface of the Sun. Radiative transfer is an exchange of thermal energy between hot bodies caused by the processes of emission, transfer, reflection, absorption of radiation energy. High energy photons collided with electrons and ions are resulting in repeated light and thermal re-emissions. By going farther from the center of the Sun, the general radiation flux distributes over the bigger area, which is proportional to the square of its radius and that's why the flux decreases per unit of area. According to StefanBoltzmann law the total amount of radiation $E$ per unit surface area is directly proportional to the fourth power of its temperature: $E=s T^{4}$, where $s$ is Stefan-Boltzmann constant. The $4 \pi r^{2} s T^{4}$ is constant and equals to the luminosity $L$ in the area of radiative transfer where energy sources are not. The temperature in middle layers of the Sun is inversely proportional to square root of distance to the center.

Inner parts of the Sun are impossible to see directly by means of conventional methods. Physical conditions inside the Sun may be determined based on theoretical calculations and checked, for example, based on studies of acoustic waves' distribution in the depth of the Sun (helioseismology). Besides the energy released during the thermonuclear reactions in the form of $\gamma$ - quanta and kinetic energy of interacting particles, it is very important to mention about the creation of neutrino the flux of which practically pierces the interplanetary space and our Earth. Another possibility to see a zone of nuclear reactions in the central areas is by means of registering solar neutrinos that are produced by nuclear reactions inside the Sun. Neutrinos are very weakly interacting particles. That's the reason why they easily escape from the solar core and with the velocity close to the light, distribute throughout the outer space without absorption.

External layers: Outside the radiation zone there is a convection zone (a layer with the thickness of 150-200 thousand $\mathrm{km}, \mathrm{f}$ ). We can see only the surface of the convection zone in the form of photosphere granulation and chromospheres' spicules. The heated flows of plasma move up, giving their energy to the upper layers and are heated up again when they are flowing down. Convective flows form separate cells of solar atmosphere (granules, fig.6a) and chromosphere cells (spicules). The intensity of plasma processes on the Sun periodically changes (11-year period of solar activity). Densities are $10^{17}-10^{18} \mathrm{~cm}^{-3}$, and temperature is 7-8 thousand degrees. The convection zone on the solar surface is manifested by next manner. The upper cells are observed in the form of granules. Deeper large-scale moves by second layers are manifest as super granulation cells and chromosphere net. There are reasons to believe that the convection in still deeper layers has in form of enormous structures or cells of even greater sizes than supergranulation. 


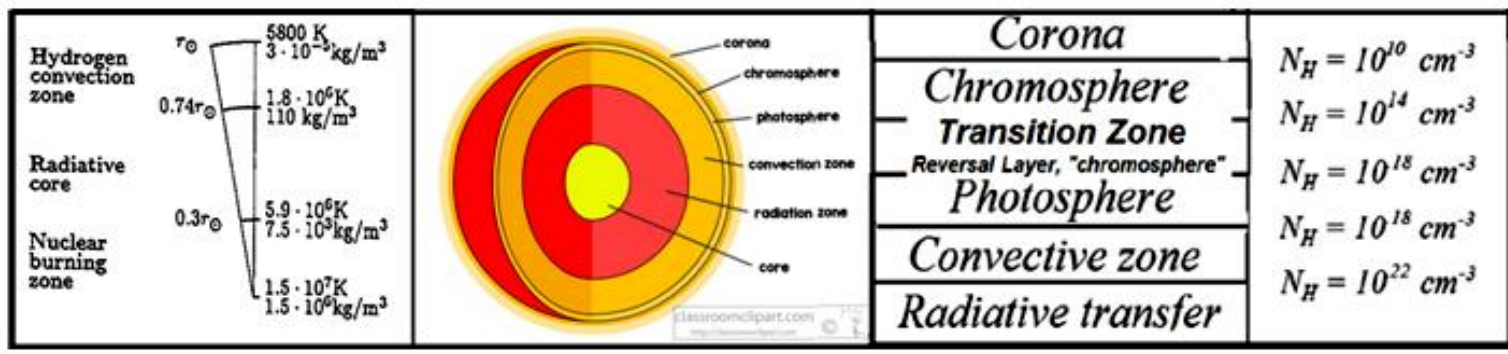

Fig.5.1. Internal and external zones of the Sun are shown here. a. Standard model of the Sun (Physical encyclopedia.mht).

$\boldsymbol{b}, \boldsymbol{c}$. External zones and their physical borders according our data.

Fig.5.2. Photosphere is the very thin sheet $(\sim 300 \mathrm{~km}) /$

Photosphere is a visible surface of the Sun. It has a thickness of $\sim 0,001 R_{\odot}(\approx 320 \mathrm{~km})$ and density of $10^{-9}-10^{-6} \mathrm{~g} / \mathrm{cm}^{3}$. It is a zone where gas layers change from completely opaque for emission to absolutely transparent. Temperature of solar photosphere is $\sim 5800{ }^{\circ} \mathrm{K}$ and it lower (to $\sim 4800{ }^{\circ} \mathrm{K}$ ) at the foot of chromosphere. Absorption lines in the spectrum of the Sun are formed as the result of emission scattering in the solar transition zone. Photosphere is the only zone that primarily consists of non-ionized atomic hydrogen. Simpler molecules and radicals of $\mathrm{H}_{2}, \mathrm{OH}$, and $\mathrm{CH}$ type are being preserved only in the

\begin{tabular}{|cccc|}
\hline \multicolumn{4}{|c|}{$\begin{array}{c}\text { Solar Photosphere as a Function of } \\
\text { csep10.phvs.utk.edu/ }\end{array}$} \\
$\begin{array}{c}\text { Depth } \\
\text { (km) }\end{array}$ & $\begin{array}{c}\text { astr162/lect/sun/photosphere.html } \\
\text { \% Light from } \\
\text { this Depth }\end{array}$ & $\begin{array}{c}\text { Temperature } \\
\text { (K) }\end{array}$ & $\begin{array}{c}\text { Pressure } \\
\text { (bars) }\end{array}$ \\
\hline 0 & 99.5 & 4465 & $6.8 \times 10^{-3}$ \\
100 & 97 & 4780 & $1.7 \times 10^{-2}$ \\
200 & 89 & 5180 & $3.9 \times 10^{-2}$ \\
250 & 80 & 5455 & $5.8 \times 10^{-2}$ \\
300 & 64 & 5840 & $8.3 \times 10^{-2}$ \\
350 & 37 & 6420 & $1.2 \times 10^{-1}$ \\
375 & 18 & 6910 & $1.4 \times 10^{-1}$ \\
400 & 4 & 7610 & $1.6 \times 10^{-1}$ \\
\hline \multicolumn{4}{|l}{} \\
Source: Fraknoi, Morison, and Wolf, Voyages through the Universe \\
\hline
\end{tabular}
upper photosphere layers. Nevertheless, the photosphere also includes free electrons that have arisen as a result of low-level hydrogen ionization and practically full ionization of metals (calcium, sodium, magnesium, iron, etc.). By joining with neutral hydrogen atoms, electrons form negative hydrogen ions. Images of photosphere granules' structure with diffraction-limited resolution $\left(0.12^{\prime \prime}\right)$ received from stratospheric observatory on 20.06.1973 (Krat at all., 1974). Negative hydrogen ion that does not found in nature plays important role in the solar atmosphere. It's a combination of proton \& 2 electrons is manifested in the thin outer layer of the coldest of the photosphere. Namely the negative hydrogen ions emit the most of visible light. This light is actively reabsorbed by the same negative ions in the thin range $\left(N_{H} \sim 810^{17} \mathrm{~cm}^{-3}\right.$; fig.4,5) along radius that result in a fast increase of plasma opacity and the solar limb seems sharp.

Transition zone is placed by author as sub-photosphere zone. This zone is transparent yet for continuous radiation, but it is un-transparent already for radiation in lines of Lyman series. This is the formation zone of Fraunhofer lines and of the huge quantity of current sheets with plasma jets upward (fig.2). The transition zone is the place of formation of the solar wind. The transition zone is the place, where the mechanisms of energy transfer from the internal layers of the Sun are changing. Here energy of the radiative transfer is changed in energy by plasma jets out lot of small current sheets in the space between the granules. Convective elements are observed as the photosphere granulation. It was the granular structure that creates the conditions of formation by the current sheets in the photosphere-chromosphere transition zone of convective structure with its distinctive field of mass plasma flows. The new concept of transitional zone is agreed well with the current sheets available in the Sun (Chapter 5) and their direct observed manifestations in the photos (fig.1.2.1) as well as with the photometry using of solar wind manifestations (fig.3.8.1).

Chromosphere («sphere of colour») is named so because of its red and violet color. It may be observed (its spicular lower parts) during full solar eclipses and it looks like as the bright circle of a grass around a black disk of the Moon, when it has closed the Sun. It's a gaseous spicular layer of the Sun's atmosphere with the thickness of 7-8 $10^{3} \mathrm{~km}$ that is situated above the photosphere and is distinguished by its significant temperature heterogeneity $\left(5-1010^{3}{ }^{\circ} \mathrm{K}\right)$. The brightness of chromosphere is much lower than that of photosphere. That is why it may be observed only during full solar eclipses. Chromosphere can be seen as a red border around the lunar disk. It has an emission spectrum consisting of bright lines. The growth temperature up to $10000 \mathrm{~K}$ is observed in the chromosphere. It has a heterogeneous structure with bright and dark areas. The smallest structural formations are called by specula's. The image of quite Sun spicula is seen in the light of red $H_{\alpha}$ hydrogen line. Spicula are observed in $H_{\alpha}$ line up to the height of $\sim 5000 \mathrm{~km}$ until $N_{H}>10^{12} \mathrm{~cm}^{-3}$. Spicula gradually dissolve in the solar corona. Groups of solar spots (AR - active regions) with magnetic field of various polarity are by set of photospheres' crossing of magnetic flux tubes. 

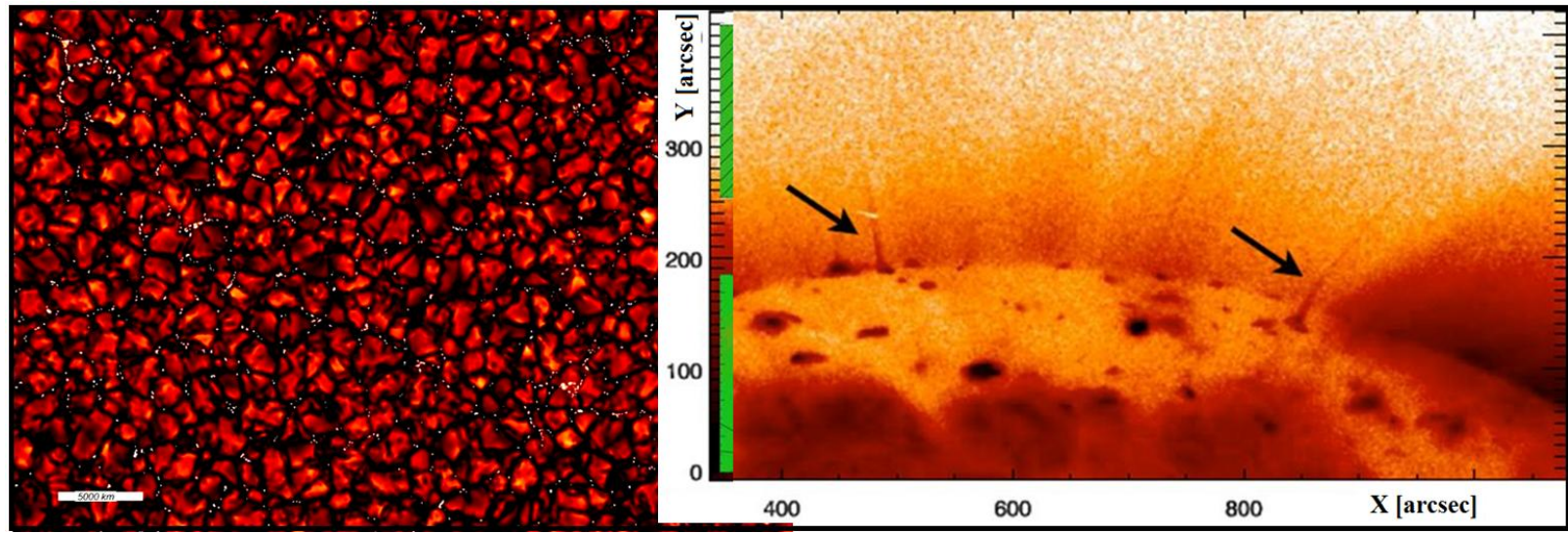

Fig.6. Plasma flows from near photosphere vertical CS and their view from the top as light points between granules (a) in the observations of the Swedish solar telescope (La Palma) and from the sidein the form of jets $(b)$ and of the Hinode.

$a$. Increase of resolution threshold opens even thinner details of granulated structure (convection zone) in optical region on the visible surface of the Sun. The bright points (their plasma jets out of vertical current sheets) are by the source of the solar wind and this is the mechanism of the heating up of the corona. The sharp, narrow-band image was recorded in 2007 using the Swedish Solar Telescope on the La Palma. Bright points are shown on the quiet Sun (APOD-16.04.2010- Sun-magnetic field-granules).

$\boldsymbol{b}$. The coronal hole on the Sun in X-ray (10.01.2007; the Hinode/XRT). Arrows indicate to two large jets of directed ejections (Müller \& Antiochos, 2008). A great number of smaller jets create continuous flow of solar wind that points to the nonthermal nature of coronal heating.

Corona is an most external and extensive zone of solar atmosphere (fig.1). Chromosphere spicules dissolve in the corona and together with the CS accelerated flows have compounded of the corona. That's the way how the exchange of matter between chromosphere and corona is happening. The corona is "heated" up to $>10^{6}{ }^{\circ} \mathrm{K}$. The nature of processes that heats the corona, supports its "high temperature" and speeds up the solar wind were of mystery until our discovery of the current sheets on the Sun. Temperature inside the Sun is got from the heat of nuclear sources is decreased further as distanced from the solar core. Temperature of nuclear reactions zone (in tens of millions degrees) is lowered (as photons are re-emitted during radiative transfer and convection) to $6000 \mathrm{~K}$ on the photosphere level. Then, on comparatively short distance by the transition zone (fig.1), the temperature starts to rise rapidly to high values. Few mechanisms of such heating up were considered. However, they cannot be substantiated in reality neither each of them separately, nor in any combination. At the same time, after discovering natural current sheets $(C S)$ that occur on the Sun (Ostapenko, 2016), the problem can be regarded as solved. The change of the mechanism of thermal transfer of radiative energy into the other non-thermal mechanism of the magnetic acceleration of plasma and charged particles occurs in current sheets in the transition zone.

Electromagnetic and corpuscular emission of the Sun (starts) is very powerful and diverse. Corpuscular component are fluxes of protons and electrons. Electromagnetic emission covers the wavelength band from radio waves up to $\gamma$-radiation. In its visible region, the spectrum is written by the Plank law of the black body radiation. The Sun (the star of G2 class, $~ 5800 \mathrm{~K}$ ) emits a maximum amount of radiation in the visible region (430-500 nm). The solar spectrum is continuous and has >20 thousand of absorption lines (Fraunhofer lines) of different elements. Continuous absorption coefficient $k, \mathrm{~cm}^{2}$ per one heavy particle in photosphere at optical depth $\tau_{5}=0.1$ on the wave $5000 \AA$ and $\log p_{e}=0.5$, depending on the wavelength $\lambda$. It arises due negative hydrogen ions excitation and no changes with temperature. Spectral lines arise on the continuous background.

\section{Discussion}

The first jets were recorded by a 1st-generation X-ray telescope onboard Skylab in the 1970s. They were called X-ray jets for the simple reason that they were bright at X-ray wavelengths. The phenomenon was later confirmed by a Naval Research Lab ultraviolet telescope that flew aboard the space Shuttle in the 1980s as well as by Japan's Yohkoh/XRT in the 1990s. All those instruments saw very few jets (one or two per day). Xray jets were thus regarded as a curiosity of little importance.

We must to note, the flare' active processes has been studied in detail by soviet scientists (Syrovatskij, 1967, 1974, 1975, and 1979). The Severny' "moustache" (or Ellerman' "bombs") one of greatest manifestations 
The Field Of Lyman-Alpha Radiation plus Current Sheets and Physics of Different Zones of the ..

of flare' activity near the photosphere were studied experimentally by Severny (1954, 1957, and 1958) in Crimea Astrophysical Observatory. Spectral modeling of compact flare' events near the photosphere are made by Ostapenko (1980). Surges, sprays, canalized flows and jets were modeling and photometrical study by Ostapenko $(1981,1985)$ as possible manifestations of the Syrovatskij theoretical current sheets (CS). All this has ended by the discovery of the theoretical current sheets in the Nature (Ostapenko, 1997, 2016) and has written in monographs by Ostapenko $(2011,2012)$. Solar flares, Severny' "moustache" and all other compact flare-like formations of transition zone (near photosphere) are proven to be current sheets directly.

Hinode has detailed all that. The spacecraft's advanced X-Ray Telescope can take pictures rapidly enough to catch these fast-moving eruptions. The jets happen all the time, as often as 240 times a day. They appear at all latitudes, within coronal holes, inside sunspot groups, out in the middle of nowhere. They are a major form of solar activity. The jets liken to the twinkle of Christmas lights, randomly oriented within a coronal hole. The X-ray jets have been seen before, but never in such abundance. Each jet is triggered by a magnetic eruption or "reconnection event"--essentially the same process that powers solar flares albeit on a much smaller scale. The energy in a typical jet is about a thousand times less than the energy of an M-class (medium sized) solar flare. Individually, jets are weak; however, they pack quite a punch. If we add up all the energy jets deposit into the Sun's atmosphere, the daily total is on par with solar flares (Jonathan Cirtain, 2007).

Astronomers using Japan's Hinode spacecraft (Jonathan Cirtain, 2007) have see that the Sun is bristling with powerful "X-ray jets." They spray out of the Sun's surface hundreds of times a day; launching blobs of hot (or accelerated due the reconnection mechanisms?) gas as wide as North America at a top speed of 10-100 10 3 $\mathrm{km} / \mathrm{s}$. These jets add significant mass to the solar wind and they may help explain a mystery of astrophysics: the superheating of the Sun's corona.

The 2006 launch of the Hinode satellite changed the picture of the Sun for astrophysicists (Scott McIntosh et al., 2006). The Hinode/XRT captures images of the Sun in detail. These new images led them to a new way of considering why the solar corona is millions of degrees hotter than the Sun's visible surface. The Hinode observes all zones of solar atmosphere from photosphere to corona. Using Hinode imagery, sciences discovered the new type of spicules. The classic type-I spicules are jets of dense plasma that shoot up from the chromosphere and, more often than not, return along the same path. The type-II spicules are hotter, shorter lived and faster moving than their type-I brethren. The radices appeared to shoot upward and disappear, often moving at speeds in $>100 \mathrm{~km} / \mathrm{sec}$. These jets as authors believed likely contain plasma that ranges in temperature from $10^{4}$ to $1-210^{6}{ }^{0} \mathrm{~K}$, and have a life span of $\sim 10-100 \mathrm{sec}$. While astrophysicists have long studied Type I spicules, it is known that the material in them does not reach typical coronal temperatures. The answer has indeed cleared with the moment of the discovery of current sheets (CS) in the Nature. These are the high-speed CS plasma jets or flows (Ostapenko, 2016) but not high-temperature plasma as have believed Scott McIntosh et al. (2006).
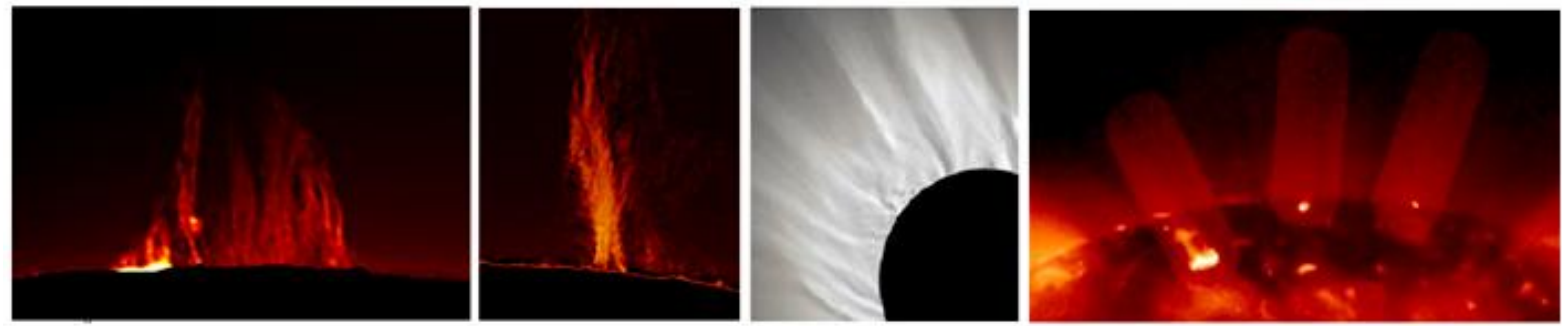

Fig.7. An X-ray jet recorded in $U V$ and $X$ [ray wavelengths.

$\boldsymbol{a}$. A Solar Prominence $\boldsymbol{b}$. A Solar Prominence $\boldsymbol{c}$. The big corona after Images in UV (Xiao Sun, Images in UV (Xiao Sun, Koen van Gorp (2007) 2010). 2010). (APOD 26.07.2009).

d. An X-ray jet recorded by the Hinode spacecraft on January 10, 2007.

The solar prominence images come also from another Spanish astrophotographer, Jesús Carmona de Argila (Xiao Sun, 2011; fig.7) with the DMK 21AF04. Astronomy camera to record these jets views for the year of 2010. During the noon hours of 30 January 2015, a coronal jet was visible near the Sun's east limb. Coronal jets are rather narrow ("squeezed") ejections of solar plasma that result from magnetic reconnection between some emerging flux and pre-existing "open" field structures. The reconnection results in jets that emit material up into the higher solar atmosphere and even into space if the eruption is strong enough. The precise mechanism (s) behind the acceleration and squeezing of the jet are not known yet in full detail. See the movie of this impressive event. The 30 January jet was visible in EUV from about 12:55 till 14:00 UT. It did not leave an obvious X-ray signature, the X-ray flux being mostly influenced by the solar flare activity from active regions NOAA 2268 and 2277. Indeed, the latter produced a short-duration M2 flare peaking at 12:16 UT, while the enhanced flux around 12:56 UT was produced by activity in NOAA 2268. The slow X-ray evolution of the latter event was not even classified as a flare. 


\section{Conclusions}

The research history of active processes on the Sun has a long period already. In relation to solar flares, this problem was formulated as the main task of International Geophysical Year (IGY-1957). But this problem was resolved after more then 50 years in result of discovery the nature' current sheets by Ostapenko V. A. in the beginning of new Millennium. The prime source of this energy is proved to be the magnetic fields. The transformation mechanism of magnetic field energy into energy and dynamics of plasma is the current sheers that was founded by Syrovatskij S. I. theoretically in the second half of $1970^{\text {th }}$.

The solution of the problem of the Layman-alpha radiation field in the solar atmosphere simultaneously with discovery of the current sheets $(C S)$ in the Nature (on the Sun) have allowed by the author to revise both the available models of solar atmosphere and the heat nature of the corona. We distinguish four zones now in the external solar atmosphere, in their sequence and in their physical sense: photosphere, chromosphere, transition zone and corona. Now, it is believed that coronal plasma temperature has exceeded of million degrees. Our results indicate the structure of the corona composed of multiple non-thermal jets of the CS that change the very notion of the term of temperature. The corona is transparent in all hydrogen lines of the Lyman series (the A case of Menzel approximation) and radiative processes take only place in excitation of hydrogen plasma.

In the solar atmosphere physical conditions of any of zones are determined, in other equal conditions, by the hydrogen plasma density only. The corona begins when the density of plasma will decreased to $n_{H}<10^{10}$ $\mathrm{cm}^{-3}$. The chromosphere occupied the plasma density diapason of $10^{10}<n_{H}<10^{14} \mathrm{~cm}^{-3}$. The transition zone occupied the plasma density diapason of $10^{14}<n_{H}<10^{18} \mathrm{~cm}^{-3}$. In this zone the LTE is achieved into all hydrogen lines of the Lyman series (the B case of the Menzel approximation) and electron impact processes take only place in excitation of hydrogen plasma. Here discrete lines of absorption are formed (the Fraunhofer spectrum of the Sun). And all conditions for the creation of multiple vertical CS with directed streams ejections take place because the plasma gradient is presence. And when the density $n_{H}>10^{18} \mathrm{~cm}^{-3}$, the continuous spectrum of the Sun arises, which is the radiation spectrum of negative hydrogen ions.

In a vertical current sheet, the plasma density gradient causes a sharp decrease in the thickness of the compressed CS layer up to zero to the its middle and it turns the CS into the piston with one-sided eject up of the plasma. Multiple set of such accelerated plasma flows up and provides seemingly "heat" of the corona.

\section{References}

[1]. Baker J. G. \& Menzel D. H. Physical processes in gaseous nebulas. III. The Balmer decrement. Astrophys. J., 1938, v.88, p.52-64.

[2]. Burch J. L., Drake J. F. Reconnecting magnetic fields. American Scientist, 2009, v.97, p.392-399.

[3]. Chultem T. \& Yakovkin N. A. Stationary equations of hydrogen excitation and ionization in prominences. Solar Phys., 1974, v.34(1), p.133-150. SAO/NASA Astrophysics Data System (ADS). http://articles.adsabs.harvard.edu//full/ ...34..133C/0000135.000.html.

[4]. Jonathan Cirtain. The Hinode X-ray jets. The Marshall Space Flight Center, Nov. 2006, http://science.nasa.gov/sciencenews/science-at-nasa/2007/06dec_xrayjets/

[5]. Koen van Gorp. NEW DISCOVERY: THE SUN IS BRISTLING WITH X-RAY JETS. HTTP://SCIENCE.NASA.GOV/SCIENCENEWS/SCIENCE-AT-NASA/2007/06DEC_XRAYJETS/

[6]. Krat V. A., Dulkin L. Z., Validov M. A., Vachrameev I.Ya., Karpinsky V. N., Muzalevsky Yu. S., Nikolaev R. P., Protsenko B. A., Sobolev V. V., Tabakova Z. N., Shachbazyan Yu. L. Fourth flight of Soviet Stratosphere' Observatory. Astron. Cirkuljza, 1974,28 Jan., No. 807, p.1-3 (ru).

[7]. Krivsky L., Kurochka L. On Lyman's emission of solar flares. Bull. Astron. Inst. Czechosl., 1974, v.25, p.52-61.

[8]. Kurochka L. N., Kurochka E. V., Ostapenko V. A. Radiation of chromospheric flares in hydrogen lines. Bull. Astron. Inst. Czechosl., 1975, v.26, p.23-29.

[9]. Kurochka L. N., Kurochka E. V., Ostapenko V. A. Electron temperature and Lyman-alpha radiation temperature in solar formations. Solnechnye dannye (Pulkovo), 1978, No.12, p.94-101.

[10]. Lei Huang \& Cong Yu. Magnetar Giant Flares in Multipolar Magnetic Fields --- II. Flux Rope Eruptions With Current Sheets. Cornell University Library, 2014. http://arxiv.org/abs/1409.4488/

[11]. Müller D. A. N., Antiochos S. K. Topologically driven coronal dynamic-a mechanism for coronal hole jets. Annales Geophysicae, 2008, v.26, p.2967-2974.

[12]. Ostapenko V. A. The probabilities of ionization and excitation of hydrogen atoms by the electron impact. Vestn. Kiev un-ty, Astronomy. 1974, No.16, p.42-54 (ru).

[13]. Остапенко B.A. Profiles of emission lines of compact chromosphere'formations. Astron. J., 1980, v.57, p.362-371 (ry). Emission line profiles of compact chromospheric formations http://www.osti.gov/scitech/biblio/5003075; Sov. Astron. AJ? 1980/03/01 (Engl. Transl.); (United States); Journal Volume: 24:2. https://www.researchgate.net/publication/234190371_Emission_Line_ Profiles_of_Compact_Chromospheric_Formations

[14]. Ostapenko V.A. On the possibility of observations of the current sheets on the Sun. Pis'ma in Astron. Journal, 1981, v.7, p.561-565 (ru). Are current sheets observable on the Sun? Soviet Astronomy Letters, v.7, 1981, p. 313-315. 2014, http://adsabs.harvard.edu/full/1981SvAL ...7..313O. https://books.google.com.ua/ books?id=tCrsCAAAQBAJ\&pg=PA239\&lpg $=\mathrm{PA} 239 \& \mathrm{dq}=$ Ostapenko $+\mathrm{V}$. A. + current + sheet $\&$ sourc...

[15]. Ostapenko V.A., Palush P., Vatsulik V. On the structure and dynamics of solar flares. Acta Astronomica et Geophysika Universitatis Comenianaex, 1985, v.10, p.3-25 (ru). 
The Field Of Lyman-Alpha Radiation plus Current Sheets and Physics of Different Zones of the ..

[16]. Ostapenko V.A. Preliminary results of optical solar flare spectra studies using a 40-channel densitometer. Ap \& space science. 1997,v.252,p.335-348.https://www.researchgate.net/publication/226894321_Preliminary_Results_of_Optical_Solar_Flare_ Spectra_Studies_Using_a_40-Channel_Densitometer

[17]. Ostapenko V. A. Solar flares: current sheets on the Sun, ecological hydrogen power on the Earth. Kiev,"Ukraine", 2011 , 450 p. (ru)

[18]. Ostapenko V.A. Solar flares - current sheets on the Sun, ecological hydrogen power on the Earth. Kiev, "Ukraine", 2012, 185 p.

[19]. Ostapenko V. A. Research programme of International Geophysical Year (IGY-1957) have ended in the Millennium beginning by discovery of the theoretical current sheets in the Nature. International Journal of Current Research, 2016, v.8, No.7, 11 p.; journalijcr_2014@yahoo.in.

[20]. Podgorny A. I. \& Podgorny I. M. The model of a solar flare in comparison of observations of high-energy processes. Astron. J., 2006, v.83, No.10, p.940-949 (ru).

[21]. Scott McIntosh, Bart De Pontieu, Viggo Hansteen and Karel Schrijv. https://ncar.ucar.edu/press/ why-is-the-suns-atmosphere-so-hot

[22]. Seaton M. J. The solution of capture-cascade equations for hydrogen. M. N. R. A. S., 1959, v.119, p.90-97.

[23]. Severny A. B. About the magnetohydrodynamic phenomena at the Sun's surface. Izv. Krymsk. astrophys. observ., 1954, v.11, p.129-151 (ru).

[24]. Severny A. B. Study on fine structure emission active entities and transient processes on the Sun. Izv. Krymsk. Astrophys. observ., 1957, v.17, p.129-161 (ru).

[25]. Severny A. B. Nonstationary processes in solar flares as a manifestation of the pinch effect Astron. J., 1958, v.35, p.335-350 (ru).

[26]. Sobolev V. M. Investigations of hydrogen and helium excitation in solar prominences. Izv. GAO in Pulkovo, 1958, No.158, p.1266. Stationary distribution of hydrogen atoms on states in atmosphere of the Sun. 1961, No.167, p.52-85 (ru).

[27]. Structure of the Sun. https://perswww.kuleuven.be/ u0052182/weather/les 1/node3.html

[28]. Syrovatskij S. I. Fast particle generation in space conditions. Izv. Of AS of Soviet Union. Ser. Phys., 1967, v.31, p.1303-1306 (ru).

[29]. Syrovatskij S. I. The process of reconnection of magnetic field lines, and its role in space plasma. Materials of VII Leningrad International 1975 seminar. "Corpuscular. Sun streams and radiance zone of the Earth and Jupiter, 1975". L., 1975, p.63-80 (ru).

[30]. Syrovatskij S. I. Current sheets and flares' processes in laboratory and space plasmas. UFN (Successes of Phys. Sciences), 1976, v.118, p.738-741. Vestnik of SU AS, 1977, v10, p.33-44 (ru).

[31]. Syrovatskij S. I. Key problems of theory of solar flares. Izv. of AS of Soviet Union. Ser. phys., 1979, v.43, No.4, p.695-707 (ru).

[32]. Xiao Sun. Solar Prominence Images, 2011; http://www.astronomycameras.com/blog/archive/20110119/ 